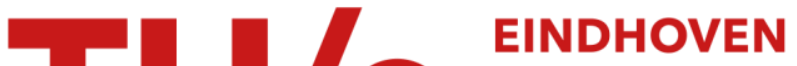

\section{Bifurcation theory for the $\mathrm{L}-\mathrm{H}$ transition in magnetically confined fusion plasmas}

Citation for published version (APA):

Weymiens, W., Blank, de, H. J., Hogeweij, G. M. D., \& Valença, de, J. C. (2012). Bifurcation theory for the L-H transition in magnetically confined fusion plasmas. Physics of Plasmas, 19(7), [072309].

https://doi.org/10.1063/1.4739227

DOI:

10.1063/1.4739227

Document status and date:

Published: 01/01/2012

\section{Document Version:}

Publisher's PDF, also known as Version of Record (includes final page, issue and volume numbers)

\section{Please check the document version of this publication:}

- A submitted manuscript is the version of the article upon submission and before peer-review. There can be important differences between the submitted version and the official published version of record. People interested in the research are advised to contact the author for the final version of the publication, or visit the $\mathrm{DOI}$ to the publisher's website.

- The final author version and the galley proof are versions of the publication after peer review.

- The final published version features the final layout of the paper including the volume, issue and page numbers.

Link to publication

\section{General rights}

Copyright and moral rights for the publications made accessible in the public portal are retained by the authors and/or other copyright owners and it is a condition of accessing publications that users recognise and abide by the legal requirements associated with these rights.

- Users may download and print one copy of any publication from the public portal for the purpose of private study or research.

- You may not further distribute the material or use it for any profit-making activity or commercial gain

- You may freely distribute the URL identifying the publication in the public portal.

If the publication is distributed under the terms of Article $25 \mathrm{fa}$ of the Dutch Copyright Act, indicated by the "Taverne" license above, please follow below link for the End User Agreement:

www.tue.nl/taverne

Take down policy

If you believe that this document breaches copyright please contact us at:

openaccess@tue.nl

providing details and we will investigate your claim. 


\title{
Bifurcation theory for the L-H transition in magnetically confined fusion plasmas
}

\author{
W. Weymiens, ${ }^{\text {a) }}$ H. J. de Blank, G. M. D. Hogeweij, and J. C. de Valença \\ FOM Institute DIFFER - Dutch Institute for Fundamental Energy Research, Association EURATOM-FOM, \\ Trilateral Euregio Cluster, P.O. Box 1207, Nieuwegein, The Netherlands
}

(Received 13 April 2012; accepted 3 July 2012; published online 23 July 2012)

The mathematical field of bifurcation theory is extended to be applicable to 1-dimensionally resolved systems of nonlinear partial differential equations, aimed at the determination of a certain specific bifurcation. This extension is needed to be able to properly analyze the bifurcations of the radial transport in magnetically confined fusion plasmas. This is of special interest when describing the transition from the low-energy-confinement state to the high-energy-confinement state of the radial transport in fusion plasmas (i.e., the L-H transition), because the nonlinear dynamical behavior during the transition corresponds to the dynamical behavior of a system containing such a specific bifurcation. This bifurcation determines how the three types (sharp, smooth, and oscillating) of observed L-H transitions are organized as function of all the parameters contained in the model. @ 2012 American Institute of Physics. [http://dx.doi.org/10.1063/1.4739227]

\section{INTRODUCTION}

The transport from the hot core to the cold edge of magnetically confined fusion plasmas, like the tokamak, determines the energy confinement and therewith the performance of the fusion reactor. This radial transport is dominated by turbulence which greatly limits the pressure build up in the core. However, under certain circumstances, the plasma reorganizes to a self-sustained state in which locally the turbulence is suppressed. This bifurcation in the radial transport arises when, for instance, the heating power exceeds a threshold value. The resulting state of the plasma is called the high-confinement-mode, or H-mode, and was first discovered by the ASDEX Team in 1982. ${ }^{1}$ The physical mechanism causing this transition from the L-mode (lowconfinement-mode) to the H-mode is not yet fully identified ${ }^{2}$ however, the nonlinear dynamics observed during the L-H transition can be identified as certain fundamental bifurcations, which give directions to the possible underlying dynamical equations. Bifurcation theory categorizes all topological changes of the solutions of dynamical systems as function of a corresponding control parameter. The L-H and H-L transitions are quite naturally characterized as fold bifurcations, which describe the creation and disappearance of stationary solutions by slight changes of the relevant parameters. This fold bifurcation then describes the sudden disappearance of the L-mode stationary state by increasing the heating power slightly above a threshold value, therefore forcing the system to sharply transit to the only stationary state left, i.e., the H-mode. Because the back transition from the $\mathrm{H}$-mode to the L-mode occurs at a different value of the heating power, there must be a separate fold bifurcation causing the disappearance of the H-mode. Thus, two separate fold bifurcations are needed to describe hysteresis like behavior. With bifurcation theory, it is proven that the existence and magnitude of the hysteresis between L-H and H-L

\footnotetext{
a)w.weymiens@differ.nl.
}

transitions can be controlled by two types of parameters. By varying the first type of parameter, the disappearance of hysteresis occurs when the two fold bifurcations meet in a so-called cusp bifurcation. Varying the second type of parameter can cause the hysteresis (two stable solutions) to be replaced by limit cycle oscillations (no stable solutions) due to a Hopf bifurcation of the system. These two different regimes where the hysteresis has disappeared are also observed in tokamak plasmas. The first one results in smooth transitions ${ }^{3}$ for both the L-H and H-L transitions without any bifurcations. The second one results in oscillatory behavior corresponding to a phase of dithering $\mathrm{H}$-mode ${ }^{2,4}$ before the system settles into the final $\mathrm{H}$-mode. How these different types of L-H transition phenomena are ordered relative to each other as function of the parameters of the model is determined by the underlying bifurcations. Indeed, hysteresis behavior in a dynamical system is governed by two separate fold bifurcations. As noted before, there are two ways of destroying this hysteresis behavior according to bifurcation theory. Correspondingly, there are two types of parameters which will do this. One leading to the cusp bifurcation at which the magnitude of the hysteresis has shrunk to zero, and the other leading to a Hopf bifurcation due to which the hysteresis is replaced by oscillations. These two separate directions in parameter space branch off out of the underlying co-dimension 3 bifurcation. With the analysis of this bifurcation, it is possible to find with which parameter or combination of parameters the system evolves towards the different transition regimes [sharp (i), smooth (ii), and oscillating (iii)], and simultaneously to determine the threshold values of these parameters or combination of parameters.

The lowest order dynamical system containing this codimension 3 bifurcation is the FitzHugh-Nagumo model ${ }^{5,6}$

$$
\dot{x}=-a-b x-x^{3}+c y, \quad \dot{y}=-x-y,
$$

which allows a clear visualization of the different types of directions for the different bifurcations and therewith the 

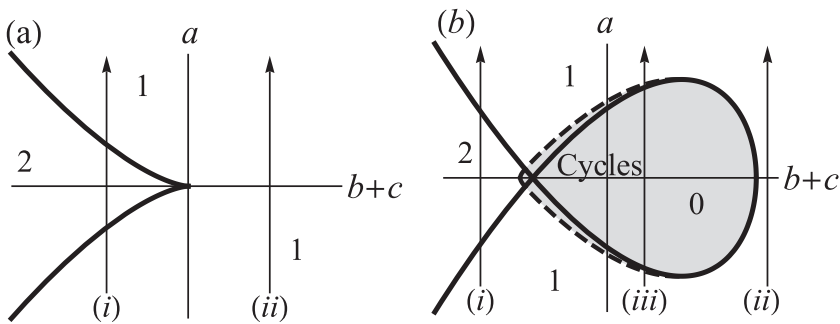

FIG. 1. (a) The fold bifurcations surrounding the region with multiple stable stationary states (which are indicated by the numbers) that merge at the cusp bifurcation at the origin of the graph. The cusp bifurcation divides the parameter space in a region $(b+c<0)$, where sharp transition with hysteresis occurs [trajectory (i)], and a region $(b+c>0)$ with smooth transitions without bifurcations [trajectory (ii)]. This structure is valid for all $c<c_{c r i t}$ in the FitzHugh-Nagumo model. (b) If $c>c_{\text {crit }}$ the cusp bifurcation gets covered with a region of limit cycle solutions, indicated by the shaded area, where the solid curve corresponds to the Hopf bifurcation and the dashed curve to a nonlocal bifurcation (described in Sec. III) both generating the limit cycles. Thus, the region to the left [containing trajectory (i) describing a sharp transition] and the region to the right [containing trajectory (ii) describing a smooth transition] are now separated by a region with oscillatory transitions [the shaded region containing trajectory (iii)].

fundamental structure of the ordering for the different types of L-H transition phenomena around a co-dimension 3 bifurcation. For $c=0$, the steady state solutions obviously can have one or multiple possibilities depending on the parameters $a$ and $b$. The number of fixed points can only be changed at the fold bifurcations of the system which determine the characteristic cusp-shaped curve plotted in Fig. 1(a) bounding the region with multiple solutions. This directly indicates that the $b$-parameter is the one which can shrink the hysteresis to vanishing size by merging the two fold bifurcations at the origin of the graph, i.e., the cusp bifurcation. For $c \neq 0$, the bifurcation structure of the system stays actually exactly the same, only until $c$ is increased above some threshold value $c_{\text {crit }}=1$ a region of limit cycle solutions will appear, i.e., the shaded region in Fig. 1(b), which covers the cusp bifurcation and its fold bifurcations branching off of it. Passing through this region of oscillations (iii), indeed, no hysteresis will be present.

Thus, knowing that this co-dimension 3 bifurcation is present in a dynamical system, it is automatically proven that this system contains sharp transitions between different stationary states which exhibit hysteresis, that there are also smooth transitions between the different states, and that there are oscillatory transitions (see Fig. 2). If a detailed model for the edge transport barrier dynamics contains such a codimension 3 bifurcation, it is clear that the regions of parameter space with L-mode, H-mode, hysteresis, and dithering are organized in the same way as the $(a, b, c)$ parameter space of system Eq. (1). The determination of these bifurcations is straightforwardly possible in coupled systems of ordinary differential equations $(\mathrm{ODEs})^{7}$ as given above. However, for the determination of bifurcations in radially resolved systems described with partial differential equations (PDEs), the bifurcation theory is strongly lacking. In Sec. II, a new analysis method is developed especially aimed at determining this co-dimension 3 bifurcation in systems of PDEs, which is, however, also applicable on the ODE cases as is shown in Sec. III. In Sec. IV, the new method is applied onto a 1-dimensional transport model for the L-H transition introduced by Zohm. ${ }^{8}$ It then becomes clear how to use this new method on radially extended dynamical models, leading to clear criteria for different types of transition behavior and the corresponding threshold values.

\section{GENERALIZED BIFURCATION THEORY}

In this section, a general method is introduced to find a co-dimension 3 bifurcation in dynamical systems specified both by PDEs and by ODEs. This is possible by realizing that one can view a system of PDEs as an infinite system of ODEs. Where one of these ODEs describes the evolution of a single point along the spatial direction coupled to its neighboring points which are separately described by their own ODE. So an infinite set of these coupled ODEs construct the entire spatial direction

$$
\dot{\mathbf{v}}=\mathbf{f}(\mathbf{v}),
$$

where $\mathbf{v}$ will be a finite dimensional vector for a set of ODEs and an infinite-dimensional vector for a set of PDEs, and $\mathbf{f}$ can be any (nonlinear) operator not incorporating spatial derivatives. The steady states of such a system are simply defined as

$$
\mathbf{f}\left(\mathbf{v}_{0}\right)=0
$$

For the co-dimension 3 bifurcation of our interest, it is sufficient to Taylor expand the system up to second order around the fixed points

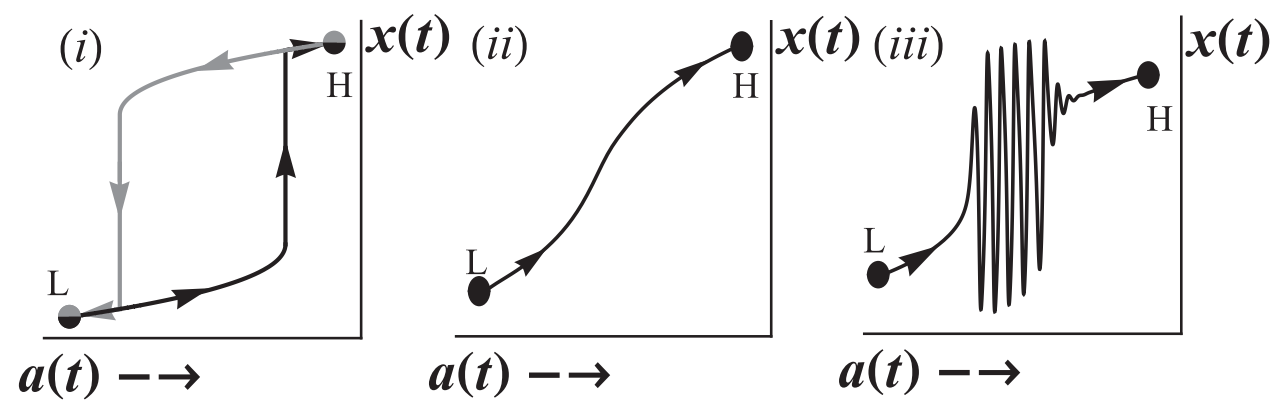

FIG. 2. The three qualitatively different transition solutions of the FitzHugh-Nagumo model for $c=3$, corresponding to the trajectories of Fig. 1 . ( $i$ ) $b=-5$, the black curve is the solution of the forward transition indicated by trajectory $(i)$. The grey curve corresponds to the inverse trajectory describing the back transition, clearly showing hysteresis. (ii) $b=0$, the solution of the evolution during a smooth transition. (iii) $b=-2.5$, with increasing control parameter $a(t)$, the system enters a regime of oscillatory solutions (limit cycles) until a second threshold is reached, after which the system settles into the other stationary state. 


$$
\dot{\mathbf{v}} \simeq \mathbf{f}\left(\mathbf{v}_{0}\right)+M_{1}\left(\mathbf{v}-\mathbf{v}_{0}\right)+\frac{1}{2}\left(M_{2}\left(\mathbf{v}-\mathbf{v}_{0}\right)\right)\left(\mathbf{v}-\mathbf{v}_{0}\right)
$$

where the linear operator $M_{1}=\partial \mathbf{f} / \partial \mathbf{v}$ (i.e., 2-tensor) and the operator $M_{2}=\partial^{2} \mathbf{f} / \partial \mathbf{v} \partial \mathbf{v}$ (i.e., 3-tensor); note that in the finite dimensional cases, the matrix multiplications are implied. If the parameters of the system are chosen such that the system is at the fold bifurcation, the linear operator $M_{1}$ becomes singular, implying a single vanishing eigenvalue with eigenvector $\mathbf{v}_{1}$. This is also true in the adjoint picture: the image space of $M_{1}$ has co-dimension 1 . Thus, at the fold bifurcation, there are vectors $\mathbf{v}_{1}$ and $\mathbf{u}_{1}$ which satisfy

$$
\begin{aligned}
& M_{1} \mathbf{v}_{1}=0, \\
& \mathbf{u}_{1}^{T} M_{1}=0,
\end{aligned}
$$

The two different possibilities of destroying the hysteresis behavior, as described in Sec. I, are caused by two different types of bifurcations: the cusp bifurcation and the Hopf bifurcation. The Hopf bifurcation arises from an underlying bifurcation, namely, the Bogdanov-Takens bifurcation ${ }^{7}$ at which the Hopf bifurcation coincides with one of the fold bifurcations. At the cusp bifurcation, both the fold bifurcations coincide; if all three bifurcations coincide (i.e., the two fold bifurcation and the Hopf bifurcation) the system is at the co-dimension 3 bifurcation. At this point, in parameter space, the system is both at the cusp bifurcation and the BogdanovTakens bifurcation at the same time, so combining the two restrictions for the cusp bifurcation and the BogdanovTakens bifurcation leads to the subspace of parameter space at which the co-dimension 3 bifurcation occurs. First of all, the cusp bifurcation is given by the point where the two fold bifurcations meet tangentially, so when the vectors $\mathbf{v}_{1}$ and $\mathbf{u}_{1}$ can be found (at the fold bifurcation) additionally the second term in the Taylor expansion [Eq. (4)] evaluated in the same direction as the fold condition must vanish,

$$
\mathbf{u}_{1}^{T}\left(M_{2} \mathbf{v}_{1}\right) \mathbf{v}_{1}=0
$$

Second, for the Bogdanov-Takens bifurcation, we need to combine the Hopf bifurcation together with the fold bifurcation. At the Hopf bifurcation, the real part of a pair of complex conjugated eigenvalues vanishes. At the fold bifurcation, the imaginary and real part of an eigenvalue vanishes. Thus, at the Bogdanov-Takens bifurcation, a pair of eigenvalues must become zero simultaneously (i.e., $\lambda^{2}=0$ ). Then, according to the Cayley-Hamilton theorem, ${ }^{9}$ there must be some generalized eigenvector, $\mathbf{v}_{2}$, and a generalized co-eigenvector, $\mathbf{u}_{2}$, satisfying

$$
M_{1}^{2} \mathbf{v}_{2}=0, \quad \mathbf{u}_{2}^{T} M_{1}^{2}=0 .
$$

At this Bogdanov-Takens bifurcation point, these generalized eigenvectors can be related to the eigenvectors which have a vanishing eigenvalue at this point, leading to

$$
M_{1} \mathbf{v}_{2}=\mathbf{v}_{1}, \quad \mathbf{u}_{2}^{T} M_{1}=\mathbf{u}_{1}^{T} .
$$

These conditions imply

$$
\mathbf{u}_{2}^{T} \cdot \mathbf{v}_{1}=\mathbf{u}_{1}^{T} \cdot \mathbf{v}_{2}
$$

The Bogdanov-Takens bifurcation will be given by the fold condition [Eq. (5)] combined with the condition

$$
\mathbf{u}_{1}^{T} \cdot \mathbf{v}_{1}=0
$$

Summarizing, if in a general dynamical system, two vectors, $\mathbf{v}_{1}$ and $\mathbf{u}_{1}$, can be found which simultaneously satisfy

$$
\begin{aligned}
M_{1} \mathbf{v}_{1} & =0, \\
\mathbf{u}_{1}^{T} M_{1} & =0, \\
\mathbf{u}_{1}^{T}\left(M_{2} \mathbf{v}_{1}\right) \mathbf{v}_{1} & =0, \\
\mathbf{u}_{1}^{T} \cdot \mathbf{v}_{1} & =0,
\end{aligned}
$$

then the system contains the co-dimension 3 bifurcation.

This analysis by itself is sufficient to conclude that the considered system has multiple steady states between which sharp transitions, smooth transitions, and oscillating transitions can occur and, moreover, that the sharp transitions exhibit hysteresis between the forward and backward transitions. However, a bit more extended bifurcation analysis might also specify for which parameter values these different types of transition take place and what the threshold values will be. For this, it is necessary to get a condition for the fold bifurcation and for the Hopf bifurcation separately. The fold bifurcation can be found quite straightforwardly by qualitatively analyzing the steady state conditions, as will become clear in Secs. III and IV where an example of a set of coupled ordinary and partial differential equations are considered, respectively. The Hopf bifurcation, however, needs some further investigation. It is already noted that the Hopf bifurcation arises out of a Bogdanov-Takens bifurcation at which there are two simultaneously vanishing eigenvalues. The Hopf bifurcation can be found by unfolding the Bogdanov-Takens bifurcation in all its parameters and by identifying the specific direction which keeps the eigenvalues purely imaginary, i.e., the Hopf condition

$$
M\left(\mathbf{v}-\mathbf{v}_{\text {new }}\right)=i \omega\left(\mathbf{v}-\mathbf{v}_{\text {new }}\right),
$$

where $M=M_{1}+\delta M$ is the linear operator of the system with the perturbed parameters. Additionally, it must be noted that the steady state solution, $\mathbf{v}_{0} \rightarrow \mathbf{v}_{\text {new }}$, is also shifted. The complex solution can be decomposed in factors of $i \omega$ where the real frequency $\omega$ is the small parameter which reduces to zero at the Bogdanov-Takens bifurcation,

$$
\mathbf{v}=\mathbf{v}_{\text {new }}+\left(\mathbf{v}_{1}+i \omega \mathbf{v}_{2}-\omega^{2} \mathbf{v}_{3}-i \omega^{3} \mathbf{v}_{4}\right) e^{i \omega t} .
$$

Consistent ordering requires that $\delta M=\omega^{2} M_{3}$, combining the same orders of $i \omega$ in the eigenvalue equation [Eq. (12)] then leads to

$$
\begin{aligned}
M_{1} \mathbf{v}_{1} & =0, \\
M_{1} \mathbf{v}_{2} & =\mathbf{v}_{1}, \\
M_{3} \mathbf{v}_{1}-M_{1} \mathbf{v}_{3} & =-\mathbf{v}_{2}, \\
M_{3} \mathbf{v}_{2}-M_{1} \mathbf{v}_{4} & =-\mathbf{v}_{3},
\end{aligned}
$$


where the first two orders give the Bogadanov-Takens point and the last two orders give the change of stability of the stationary states in the neighborhood of the Bogdanov-Takens point for small $\omega$, i.e., the Hopf bifurcation. To avoid determining the vectors $\mathbf{v}_{3}$ and $\mathbf{v}_{4}$, these equations can be dotted into the adjoint vectors $\mathbf{u}_{1}^{T}$ and $\mathbf{u}_{2}^{T}$, while using the BogdanovTakens properties of $M_{1}$, and smartly combining them leaves us with a single condition for the Hopf bifurcation that is invariant under renormalizations of the perturbation operator $M_{3}$,

$$
\mathbf{u}_{1}^{T} \cdot \mathbf{v}_{2}\left(\mathbf{u}_{1}^{T} M_{3} \mathbf{v}_{2}+\mathbf{u}_{2}^{T} M_{3} \mathbf{v}_{1}\right)=\mathbf{u}_{2}^{T} \cdot \mathbf{v}_{2}\left(\mathbf{u}_{1}^{T} M_{3} \mathbf{v}_{1}\right) .
$$

Note that all expressions, including this result, are invariant under transformations,

$$
\begin{aligned}
& \mathbf{u}_{2} \rightarrow \mathbf{u}_{2}+\lambda_{u} \mathbf{u}_{1}, \quad\left(\mathbf{u}_{1}, \mathbf{u}_{2}\right) \rightarrow \kappa_{u}\left(\mathbf{u}_{1}, \mathbf{u}_{2}\right), \\
& \mathbf{v}_{2} \rightarrow \mathbf{v}_{2}+\lambda_{v} \mathbf{v}_{1}, \quad\left(\mathbf{v}_{1}, \mathbf{v}_{2}\right) \rightarrow \kappa_{v}\left(\mathbf{v}_{1}, \mathbf{v}_{2}\right) .
\end{aligned}
$$

In Sec. III, this new analysis is proved to work properly for the already known bifurcations of a set of ordinary differential equations which contain the co-dimension 3 bifurcation. Thereafter, the method can reliably be used for the bifurcation analysis of a 1-dimensional transport model proposed in 1994 by Zohm ${ }^{8}$ to explain the dithering behavior during $\mathrm{L}-\mathrm{H}$ transitions.

\section{FINITE DIMENSIONAL CASE}

To test the new general method for finding the codimension 3 bifurcation in a set of coupled dynamical equations, it will now be applied on an already known finite dimensional example of a system containing this bifurcation. Moreover, it helps to get a feeling for the abstract machinery developed in Sec. II. As introduced in Sec. I, the FitzHughNagumo model [Eq. (1)] is such a finite dimensional model which contains the co-dimension 3 bifurcation. The steady states are straightforwardly found to be satisfying $y_{0}=-x_{0}$ and $x_{0}^{3}+(b+c) x_{0}+a=0$. Taylor expanding this system up to second order around these stationary state solutions leads to a linear operator

$$
M_{1}=\left(\begin{array}{cc}
-3 x_{0}^{2}-b & c \\
-1 & -1
\end{array}\right) .
$$

The corresponding eigenvector with vanishing eigenvalue and its adjoint can be given by

$$
\mathbf{v}_{1}=\left(\begin{array}{c}
1 \\
-1
\end{array}\right) \quad \text { and } \quad \mathbf{u}_{1}=\left(\begin{array}{l}
1 \\
c
\end{array}\right)
$$

as long as the fold condition is satisfied,

$$
\operatorname{det} M_{1}=3 x_{0}^{2}+b+c=0,
$$

which corresponds to a surface in the 3-dimensional parameter space given by

$$
a^{2}=-\frac{4}{27}(b+c)^{3} .
$$

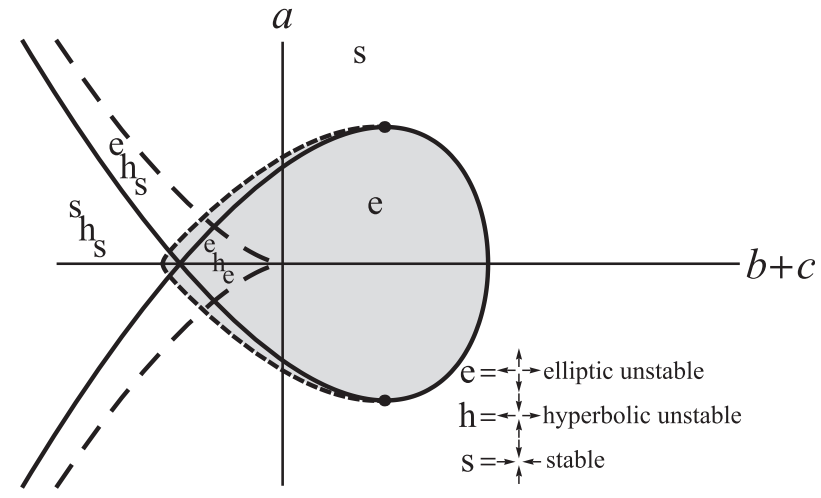

FIG. 3. 2D parameter space for fixed $c>1$ with two fold bifurcations (long-dashed curves) merging into a cusp bifurcation at $a=b+c=0$, and a Hopf bifurcation indicated by the solid curve. The short-dashed curve, corresponding to a nonlocal bifurcation, ends at Bautin bifurcations ${ }^{7}$ (black dots). The shaded area corresponds to the parameter range where stable limit cycle solutions of the system exist.

This indeed leads to the characteristic cusp shaped curves which are plotted in Fig. 3 with the long dashed lines. For the cusp bifurcation, where the fold bifurcations come together, it is necessary to consider the second order term consisting of the 3-tensor $M_{2}$; in this case, however, seven of the eight components are zero and only the $\delta x^{2}$-component is nonzero, leading to a simple restriction for the cusp bifurcation,

$$
\mathbf{u}_{1}^{T}\left(M_{2} \mathbf{v}_{1}\right) \mathbf{v}_{1}=-6 x_{0}=0 .
$$

Combining this with the fold condition leads to a line in parameter space given by

$$
b_{\text {cusp }}=-c_{\text {cusp }} \quad \text { and } \quad a_{\text {cusp }}=0 .
$$

The Bogdanov-Takens bifurcation can straightforwardly be found to be

$$
\mathbf{u}_{1}^{T} \cdot \mathbf{v}_{1}=1-c=0,
$$

leading to the Bogdanov-Takens curve in parameter space given by

$$
c_{B T}=1 \quad \text { and } \quad a_{B T}^{2}=-\frac{4}{27}\left(b_{B T}+1\right)^{3} .
$$

The intersection of these two lines gives the point in parameter space at which the co-dimension 3 bifurcation occurs,

$$
(a, b, c)=(0,-1,1) .
$$

In conclusion, this model indeed has the dynamical behavior corresponding to a co-dimension 3 bifurcation, which is ordered according to the description in Sec. I. The fold bifurcations are already found to have their characteristic shape. However, it would be nice if this match can also be done for the Hopf bifurcation. For the determination of the Hopf bifurcation, it is necessary to unfold the parameters around the Bogdanov-Takens bifurcation in the direction which keeps the eigenvalues purely imaginary, this leads to the following perturbed linear operator: 


$$
M=M_{1}+\delta M=\left(\begin{array}{cc}
-3 x_{\text {new }}^{2}-b_{B T}-\delta b c_{B T}+\delta c \\
-1 & -1
\end{array}\right) .
$$

Subtracting the linear operator evaluated at the Bogdanov-Takens point, $M_{1}$, leaves the perturbation of the linear operator to be

$$
\omega^{2} M_{3}=\delta M=\left(\begin{array}{cc}
-3 x_{\text {new }}^{2}-\left(b_{B T}+\delta b\right)-1 & \delta c \\
-1 & -1
\end{array}\right) .
$$

And $x_{n e w}$ is the new shifted fixed point, satisfying

$$
-\left(a_{B T}+\delta a\right)-\left(b_{B T}+\delta b+c_{B T}+\delta c\right) x_{\text {new }}-x_{\text {new }}^{3}=0 .
$$

This only leaves the determination of the generalized eigenvectors, $\mathbf{v}_{2}$ and $\mathbf{u}_{2}$, before the general Hopf criterium [Eq. (15)] can be used. Choose them to be

$$
\mathbf{v}_{2}=\left(\begin{array}{c}
2 \\
-1
\end{array}\right) \quad \text { and } \quad \mathbf{u}_{2}=\left(\begin{array}{l}
2 \\
1
\end{array}\right)
$$

such that the Hopf bifurcation restriction becomes

$$
-3 x_{\text {new }}^{2}-\left(b_{B T}+\delta b\right)-1=0,
$$

which leads to a surface in parameter space directly in terms of the Bogdanov-Takens values plus their perturbation. Since, this analysis is true for an arbitrarily sized perturbation, it is allowed to redefine the shifted parameters as the original parameters (e.g., $a_{B T}+\delta a=a$ ) such that the Hopf bifurcation is described by the following expression:

$$
a^{2}=-\frac{4}{27}(b+1)\left(b+\frac{3}{2} c-\frac{1}{2}\right)^{2}
$$

which is shown as a solid curve in Fig. 3. In the region of parameter space surrounded by the Hopf bifurcation, there are no stable fixed points and there is one stable limit cycle causing the oscillatory behavior. Such a region only exists for $c>c_{c r i t}=1$ and its size increases with $c$. This indeed corresponds to the co-dimension 3 bifurcation from which this Hopf bifurcation must branch off, as was expected.

Further bifurcation analysis reveals a tiny region of parameter space outside the Hopf bifurcation curve where there are still stable limit cycle solutions (i.e., in the shaded region) of the system surrounded by the short-dashed curve in Fig. 3. These cannot be found by the local analysis described in Secs. I-III, where a Taylor expansion around the fixed points is used. This global bifurcation appears when a pair of limit cycles gets created (one stable and one unstable) and separate from each other. This does not happen locally around a fixed point, but globally along the entire contour of both limit cycles simultaneously. Importantly is to note that also this bifurcation is a characteristic of the codimension 3 bifurcation, so even though the exact boundary of this global bifurcation is probably impossible to determine in an infinite dimensional system of equations, it will still enlarge the region of stable limit cycles in that case.

\section{TRANSPORT MODEL FOR THE L-H TRANSITION}

The radial transport in a fully ionized fusion plasma from the hot core towards the cold edge can effectively be described by a continuity equation for the density and the energy

$$
\begin{gathered}
\frac{\partial n}{\partial t}=-\frac{\partial \Gamma}{\partial r} \\
\frac{\partial}{\partial t}\left(\frac{n T}{\gamma-1}\right)=-\frac{\partial q}{\partial r},
\end{gathered}
$$

for this it is assumed that the transport barrier occurs in a thin layer at the edge such that a slab geometry description is allowed. Furthermore, a single temperature description is used, $T_{i}=T_{e}=T$, and it is assumed that all the particle and energy deposition into the plasma is somewhere in the core outside our modeled domain, such that there are no sources inside the domain and all the particles and energy enter as a flux which is fixed by the boundary conditions. Inside the domain, the particle and heat flux are described by

$$
\begin{aligned}
& \Gamma=-D \frac{\partial n}{\partial r}, \\
& q=-\chi n \frac{\partial T}{\partial r}+\frac{\Gamma T}{\gamma-1} .
\end{aligned}
$$

The particle flux, $\Gamma$, is governed by some effective particle diffusion due to the anomalous transport of electrons and ions. The heat flux, $q$, is a combination of some effective heat diffusion and heat advection due to the net flow described by the particle flux, with $\gamma$ the adiabatic index. A change from low confinement to high confinement can, therefore, be described by a reduction of the transport coefficients: particle diffusivity, $D$, and heat conductivity $\chi$. The exact mechanism reducing the anomalous transport coefficients is not yet known; however, some theories ${ }^{10-12}$ and experiments ${ }^{13,14}$ suggest that flows in the plasma can tear apart turbulent eddies reducing the radial extent of the transported particles and heat. In the transport model used in this paper, only a mean flow due to $\mathbf{E} \times \mathbf{B}$-drift is used, such that the transport coefficients become a direct function of the normalized radial electric field,

$$
Z=\frac{\rho_{p} e E_{r}}{T_{i}},
$$

as is shown in Fig. 4.

To describe the dynamics during possible transitions of the transport, the evolution of the radial electric field ${ }^{8,15,16}$ must be taken into account, via Ampères law which balances temporal changes of the radial electric field with the radial currents,

$$
\varepsilon \frac{\partial Z}{\partial t}=\mu \frac{\partial^{2} Z}{\partial r^{2}}+c_{n} \frac{T}{n^{2}} \frac{\partial n}{\partial r}+\frac{c_{T}}{n} \frac{\partial T}{\partial r}-G(Z),
$$

where $\varepsilon=B_{p}^{2} /\left(B^{2} \nu_{i}\right)$ is the dielectric constant of the polarized plasma. The radial currents are caused by the anomalous shear viscosity of the $\mathbf{E} \times \mathbf{B}$-drift ${ }^{17}$ (first term of right-hand 


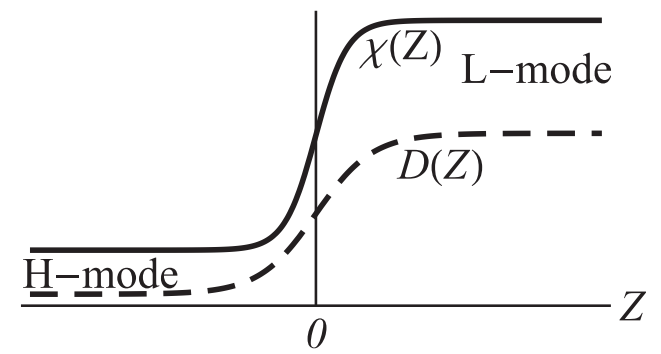

FIG. 4. The dependence of the transport coefficients (particle diffusivity, $D$, and heat conductivity, $\chi$ ) on the normalized radial electric field, $Z$. Neither the exact shape of the curve is important, nor the relative sizes of the minima and maxima as long as there is some significant difference between the minimum and maximum values, and the transition occurs for both transport coefficients around the same value of $Z$, which is chosen to be zero but can be shifted towards any other value.

side (RHS) where $\mu \sim \rho_{p}^{2}$ is the ratio of viscosity to collision frequency). The second and third terms are due to the bipolar part of the anomalous cross field flux, i.e., the excess flux of electrons relative to that of ions. ${ }^{18}$ Furthermore, radial currents can be generated due to multiple different mechanisms ${ }^{17,18}$ which depend on the radial electric field itself, e.g., ion orbit losses, bulk viscosity (due to the inhomogeneity of the magnetic field), Reynolds stress, collisional processes (e.g., ripple diffusion, gyro viscosity), charge exchange, external current drive, etc., resulting in a function of the radial electric field with many terms. However, the cusp bifurcation of the radial electric field and the corresponding transition behavior in its neighborhood in parameter space can only occur around an inflection point of this function. Therefore, it is sufficient to Taylor expand this function of many terms around its inflection point, because the characteristic transition behavior described in Sec. I will occur right there. This leads to an effective description of the last term of the electric field evolution equation

$$
G(Z)=a+b Z+Z^{3} .
$$

This closed set of coupled partial differential equations [Eqs. (32a), (32b), and (35)] is evaluated on a spatial region which must be considerably larger than the size of the transport barrier to exclude boundary effects, but small enough that the core boundary stays away from the particle and heat sources in the core of the plasma. The outer edge of the plasma at the scrape-off layer (SOL) side is fixed at $r=0$. The inner boundary of the considered spatial region is located at $r=-\infty$, this is allowed because compared to the size of the transport barrier the inner boundary is far enough away. Due to the absence of sources in the considered region, the total amount of particles and heat enters as a constant flux at this inner boundary

$$
\begin{aligned}
& \Gamma(r=-\infty)=\text { constant }=\Gamma_{-\infty}, \\
& q(r=-\infty)=\text { constant }=q_{-\infty} .
\end{aligned}
$$

At the other boundary of the system, i.e., the outer edge of the plasma, the temperature, and the density are forced to drop toward zero with a certain e-folding length into the scrape-off layer,

$$
\frac{T_{e}^{\prime}}{T_{e}}=\frac{-1}{\lambda_{T}} \quad \text { and } \quad \frac{n_{e}^{\prime}}{n_{e}}=\frac{-1}{\lambda_{n}},
$$

with constant gradient lengths $\lambda_{T}$ and $\lambda_{n}$, and where from now on the subscript " $e$ " is used for SOL edge values and primes will denote total derivatives with respect to space. Due to the absence of sources, the continuity equations can be integrated such that the steady state particle- and heat-fluxes are constant over the entire spatial domain $\left(q^{\prime}=0\right.$ and $\Gamma^{\prime}=0$, since we assume slab geometry) and can, therefore, be matched to the fluxes coming from the core,

$$
\begin{aligned}
& 0=-\Gamma_{-\infty}-D\left(Z_{0}\right) n_{0}^{\prime}, \\
& 0=-q_{-\infty}-\chi\left(Z_{0}\right) n_{0} T_{0}^{\prime}+\frac{T_{0}}{\gamma-1} \Gamma_{-\infty}, \\
& 0=-G\left(Z_{0}\right)+c_{n} \frac{T_{0} n_{0}^{\prime}}{n_{0}^{2}}+c_{T} \frac{T_{0}^{\prime}}{n_{0}}+\mu Z_{0}^{\prime \prime},
\end{aligned}
$$

where the steady state solutions are indicated with a subscript " 0 ". Because it is not expected that the L-H transition behavior is initiated by some specific difference between the transport coefficients, it is not necessary to keep track of them separately. So, without the loss of generality, it is allowed to make the following assumption on the transport coefficients:

$$
\chi(Z)=\frac{D(Z)}{\zeta(\gamma-1)} .
$$

This assumption allows us to solve the steady state density and temperature profiles as a function of the particle diffusivity alone,

$$
\begin{gathered}
n_{0}^{\prime}(r)=-\frac{\Gamma_{-\infty}}{D}, \\
n_{0}(r)=n_{0 e}-\Gamma_{-\infty} \int_{0}^{r} \frac{d r}{D}, \\
T_{0}^{\prime}(r)=\frac{\zeta D\left(Z_{e}\right)}{\lambda_{n} D}\left(T_{0 e}-T_{-\infty}\right) \hat{n}^{-1-\zeta}, \\
T_{0}(r)=T_{-\infty}+\left(T_{0 e}-T_{-\infty}\right) \hat{n}^{-\zeta},
\end{gathered}
$$

with

$$
\begin{aligned}
\hat{n} & \equiv \frac{n_{0}}{n_{0 e}}, & n_{0 e} & =\frac{\Gamma_{-\infty} \lambda_{n}}{D\left(Z_{e}\right)}, \\
T_{0 e} & =\frac{T_{-\infty}}{1+\frac{\lambda_{n}}{\zeta \lambda_{T}}}, & T_{-\infty} & =(\gamma-1) \frac{q_{-\infty}}{\Gamma_{-\infty}},
\end{aligned}
$$

where the functions of the radial electric field, like $D$ and $G$, are always functions of the steady state profile, $Z_{0}$, because deviations from steady state of those functions will always be written as a Taylor-expansion, e.g., $D(Z) \approx D\left(Z_{0}\right)+D_{Z} Z_{1}$. So, only the steady state profile of the radial electric field is needed, because the resulting diffusivity profile leads straightforwardly to the density and temperature profiles. 
Thus, consider the steady state equation for the radial electric field

$$
\begin{aligned}
0 & =G-c_{T} \frac{T_{0}{ }^{\prime}}{n_{0}}-c_{n} \frac{T_{0} n_{0}{ }^{\prime}}{n_{0}^{2}}, \\
& =G-\frac{T_{-\infty} n_{0}^{\prime}}{n_{0}^{2}}\left(c_{n}+c_{g} \hat{n}^{-\zeta}\right), \quad c_{g} \equiv \frac{\zeta c_{T}-c_{n}}{1+\zeta \frac{\lambda_{T}}{\lambda_{n}}},
\end{aligned}
$$

where the $\mu$-term is neglected. This simplification is allowed by realizing that the radial electric field jumps from a L-mode value [positive root of Eq. (43)] at the core side to a H-mode value [negative root of Eq. (43)] inside the transport barrier. This instantaneous jump (for $\mu=0$ ) is only smeared out due to this extra second derivative term (for $\mu \neq 0$ ) in a region of the order of $\mu \ll 1$. Thus, in the majority of the plasma, the value of the radial electric field is determined by the roots of Eq. (43) which are given by

$$
-G(Z) D(Z)=\frac{T_{-\infty} D\left(Z_{e}\right)^{2}}{\Gamma_{-\infty} \lambda_{n}^{2}}\left(c_{n} \hat{n}^{-2}+c_{g} \hat{n}^{-\zeta-2}\right),
$$

where the left-hand side (LHS) is a pure function of the radial electric field and the RHS is a pure function of the density, and the density is a smooth monotonic function of the radius starting from a constant $n_{0 e}$ at $r=0$ and increasing to infinity at $r=-\infty$. Therefore the RHS of Eq. (44) (and therefore also the product $-G D$ ) is a smooth monotonic function of the radius too, growing from zero at the core boundary to a constant value at the edge given by

$$
-G\left(Z_{e}\right) D\left(Z_{e}\right)=\left(c_{n}+c_{g}\right) \frac{(\gamma-1) q_{-\infty}}{\Gamma_{-\infty}^{2} \lambda_{n}^{2}} D\left(Z_{e}\right)^{2} .
$$

This restriction of the edge radial electric field corresponds to the intersection of the tilted dashed line with the curved solid line plotted in Figure 5.

From this figure, it can be clearly seen that the system can have high diffusivity (L-mode transport) or low diffusivity (H-mode transport) at the edge of the plasma depending on the slope of the tilted dashed line, $\theta$,

$$
M_{1}=\frac{\partial \mathbf{f}}{\partial \mathbf{v}}=\left(\begin{array}{c}
\nabla D \nabla \\
\nabla D T_{0}^{\prime}+\nabla D T_{0} \nabla \\
-2 c_{n} \frac{T_{0} n_{0}^{\prime}}{n_{0}^{3}}-c_{T} \frac{T_{0}^{\prime}}{n_{0}^{2}}+\frac{c_{T}}{n_{0}^{2}} \nabla
\end{array}\right.
$$

with this it is possible to construct the eigenfunctions which satisfy $M_{1} \mathbf{v}_{\mathbf{1}}=0$ where $\mathbf{v}_{\mathbf{1}}=\left(v_{n}, v_{T}, v_{Z}\right)^{T}$ together with the co-eigenvector, $\mathbf{u}_{\mathbf{1}}=\left(u_{n}, u_{T}, u_{Z}\right)^{T}$, satisfying $\mathbf{u}_{1}^{T} M_{1}=0$ and therewith find the fold bifurcation of the system. Doing the whole analysis, it turns out that the existence condition for $\mathbf{v}_{1}$ and $\mathbf{u}_{\mathbf{1}}$ reduces to a condition on the edge values. This reduction exists because the conservation laws for energy and density in steady state have a particle and heat flux

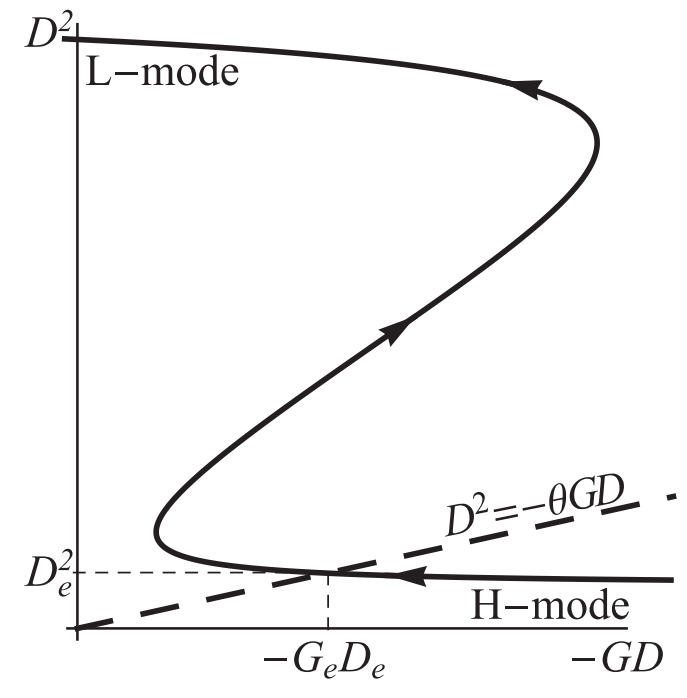

FIG. 5. The steady state solution at the edge of the plasma, $Z_{e}$, is determined by the intersection of the solid curve (where the arrows indicate the monotonic increase of $Z$ ) with the tilted dashed line, as is dictated by Eq. (45).

$$
\theta=\frac{\Gamma_{-\infty}^{2} \lambda_{n}^{2}}{q_{-\infty}(\gamma-1)} \frac{1}{\left(c_{n}+c_{g}\right)}
$$

This parameter combines most of the control parameters which can move the system into and out of the H-mode. The intersection in Fig. 5 defined in Eq. (45) determines the edge value of the radial electric field, $Z_{e}$. Combining this with the knowledge about the monotonic function of the radius $-G D$ gives the steady state profile for $Z$ and, therefore, also for $D$ such that we can use Eqs. (41b) and (41d) to produce the steady state profiles for the density and temperature of this system. As is already suspected from Fig. 5, there will be different types of solutions for different values of the parameter $\theta$. To find the transition behavior and its threshold values, the bifurcation analysis of Sec. III is applied to this set of PDEs.

For the bifurcation analysis, it is necessary to Taylor expand the system to second order around the steady states which we just found. The linear operator becomes

$$
\left.\begin{array}{cc}
0 & \nabla n_{0}^{\prime} D_{Z} \\
\nabla D n_{0}^{\prime}+\nabla D n_{0} \nabla & \nabla\left(n_{0} T_{0}^{\prime}+n_{0}^{\prime} T_{0}\right) D_{Z} \\
c_{n} \frac{n_{0}^{\prime}}{n_{0}^{2}}+\frac{c_{T}}{n_{0}} \nabla & -G_{Z}\left(+\mu \nabla^{2}\right)
\end{array}\right),
$$

which is constant over the whole radial domain, which are, therefore, equal to their boundary values. The perturbed system can also be integrated which leads to the integration constants, $\Gamma_{1}$ and $q_{1}$; however, if these would be nonzero, they would also add to the particle and heat flux at the edge, which is not allowed because these nonzero perturbations do not satisfy the boundary conditions. The perturbed system is, therefore, restricted to integration constants $\Gamma_{1}=q_{1}=0$ 
such that the boundary values of the eigenfunctions, $v_{n}, v_{T}$, and $v_{Z}$, will restrict the eventual profile of the perturbation, similar to what was possible for the steady states. Eventually, it turns out that it is only possible to find the eigenfunctions with a vanishing eigenvalue if and only if

$$
\left.\frac{d}{d Z}\left(\frac{G}{D}\right)\right|_{e}=0 .
$$

This can indeed be recognized visually in the edge steady state condition for the radial electric field (plotted in Fig. 5), as is shown by the threshold values of $\theta$ in Fig. 6(a).

The cusp condition is defined as $\mathbf{u}_{1}^{T}\left(M_{2} \mathbf{v}_{1}\right) \mathbf{v}_{1}=0$; the 3-tensor $M_{2}=\partial M_{1} / \partial \mathbf{v}_{\mathbf{0}}$ can straightforwardly be found by differentiating the first order operator $M_{1}$ with respect to $\mathbf{v}$ and then contracting it twice with the eigenvector $\mathbf{v}_{\mathbf{1}}$ after which the inner product must be taken with the co-eigenvector $\mathbf{u}_{\mathbf{1}}$. This leads again to an expression of the radial electric field at the edge as is visualized in Fig. 6(b), at which the two fold bifurcations merge together,

$$
\left.\frac{d^{2}}{d Z^{2}}\left(\frac{G}{D}\right)\right|_{e}=0
$$

For the determination of the co-dimension 3 bifurcation, it is additionally necessary to find the Bogdanov-Takens bifurcation by checking for which parameters the following condition is satisfied:

$$
\int_{-\infty}^{0}\left(u_{n} v_{n}+u_{T} v_{T}+u_{Z} v_{Z}\right) d x=0
$$

Combining the fold condition, the cusp condition, and the Bogdanov-Takens condition leads to the point in parameters space corresponding to the co-dimension 3 bifurcation

$$
(a, b, \theta)=(0,0, \infty)
$$

Thus, it is now proven that this system contains all the transition dynamics which are related to this co-dimension 3 bifurcation, i.e., sharp, smooth and oscillating transitions of the radial transport. It would also be very useful to be able to find the exact parameter space of where these different types
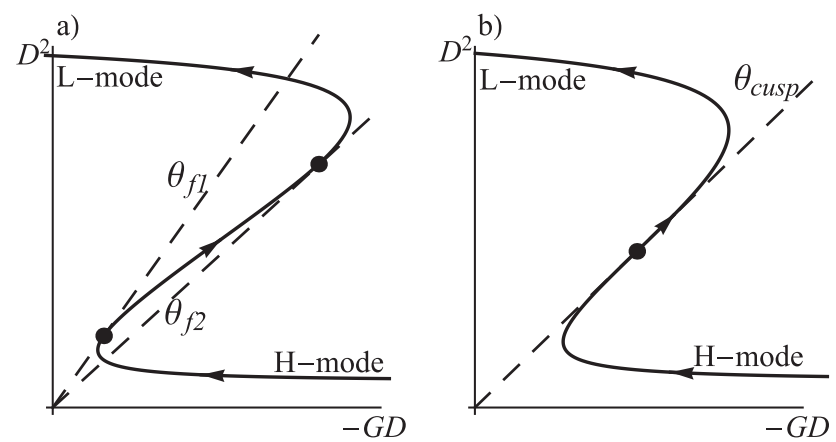

FIG. 6. (a) The visual representation of the fold bifurcation, Eq. (48), giving the threshold values $\left(\theta_{f 1}\right.$ and $\left.\theta_{f 2}\right)$ of the control parameter. (b) The two fold bifurcations merge into a cusp bifurcation $\left(\theta_{f 1}=\theta_{f 2}=\theta_{\text {cusp }}\right)$ by slight changes in the nonlinear function $G(Z)$ [e.g., the $b$-parameter of Eq. (36)] until the cusp condition is satisfied [see Eq. (49)]. of transitions may occur. For that it is necessary to find a separate condition for the Hopf bifurcations as was argued at the end of Sec. II. Following the expression of Eq. (15), the generalized eigenvector $\mathbf{v}_{\mathbf{2}}$ and generalized co-eigenvector $\mathbf{u}_{\mathbf{2}}$ are needed. These can be found by solving Eq. (8). This system can be integrated leading to integration constants, $\Gamma_{2}$ and $q_{2}$, which depend on the normal eigenfunctions $v_{n}$ and $v_{T}$,

$$
\begin{gathered}
\Gamma_{2}=\int_{-\infty}^{0} v_{n} d r \\
q_{2}=\int_{-\infty}^{0} \frac{T_{0} v_{n}+n_{0} v_{T}}{\gamma-1} d r .
\end{gathered}
$$

For $M_{3}$ the linear operator $M_{1}$ needs to be perturbed in all its parameters leading to an enormous matrix which must be contracted multiple times with different eigenvectors to generate the right Hopf criterium. This is possible, however, the extensive algebra is omitted here and replaced by a less formal analysis of guessing when the steady state profiles will destabilize, eventually ending up with the same result. From the steady state restriction for the radial electric field of Eq. (44), it was already noted that the product of functions $-G(Z) D(Z)$ will always be a monotonic function of the radius. However, if the slope $\theta$ is decreased from L-mode values towards $\mathrm{H}$-mode values, at a certain point just before reaching the fold bifurcation $-G D$ will change from increasing to decreasing at the edge. Because $Z$ is a continuous function of the radius the profile of $D^{2}$ is found by following the solid curve along the direction of the arrows in Fig. 5. This means that for those values of $\theta$ also the function $-G D$ must first increase when going inwards before it will decrease again. However, this was not allowed because $-G D$ must be monotonous, which indicates that the steady state Lmode profile must turn unstable already before the fold bifurcation is reached at those points where

$$
\left.\frac{d}{d Z}(G D)\right|_{e}=0
$$

This indeed leads to the required Hopf bifurcation condition. The same reasoning can be applied for the back transition; both transitions are plotted in Fig. 7(a).

It can be noted from Fig. 7 that the eventual transition behavior does qualitatively change when the order changes in which the bifurcations are encountered when decreasing $\theta$. For example, in Fig. 7(a) at $\theta_{H-L}$, the H-mode becomes stable due to the Hopf bifurcation of that stationary state; so by the time $\theta_{L-H}$ is reached and the L-mode turns unstable, the system will rapidly transit to the stable H-mode. The opposite holds for the back transition, when increasing $\theta$ first, the L-mode becomes stable again, and when $\theta_{H-L}$ is reached, the system rapidly jumps towards the stable L-mode. Both transitions occur at different values of the control parameter $\theta$ leading to hysteresis behavior. If another type of parameter is changed the two Hopf bifurcations might change order, leading to Fig. 7(b) where there are still sharp transition only without the hysteresis. Until the Hopf bifurcations are ordered as in Fig. 7(c), where the L-mode already becomes 

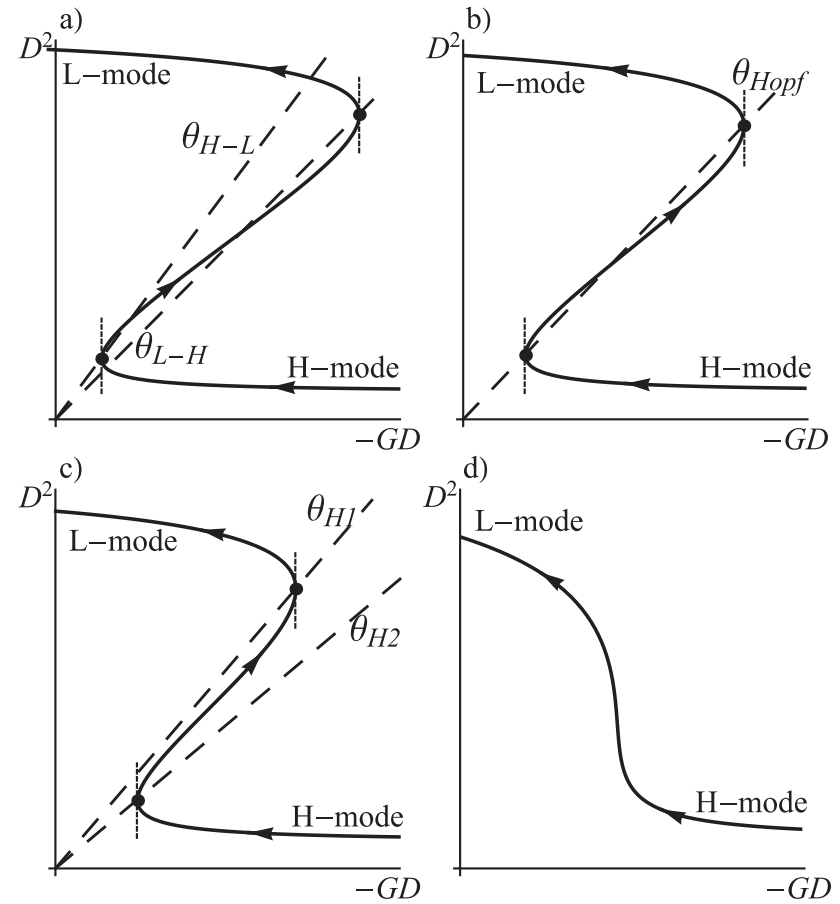

FIG. 7. The Hopf bifurcations of the edge stationary states in four different cases.

unstable while the H-mode is also unstable. At those points in parameter space where this holds, the edge stationary state cannot be on a stable fixed point but will be on a stable limit cycle solution of the system. So this will be the parameter regime of this model where the dithering solutions are situated. The same type of parameter can be changed even further such that both Hopf bifurcations merge and disappear such that there are only smooth transitions left as is indicated in Fig. 7(d). These four different types of transitions can be indicated in a complete parameter scan of this type of parameter (for this model for instance the $b$-parameter). As can be seen from Fig. 8, this parameter space is qualitatively similar to the parameter space of the FitzHugh-Nagumo model (see

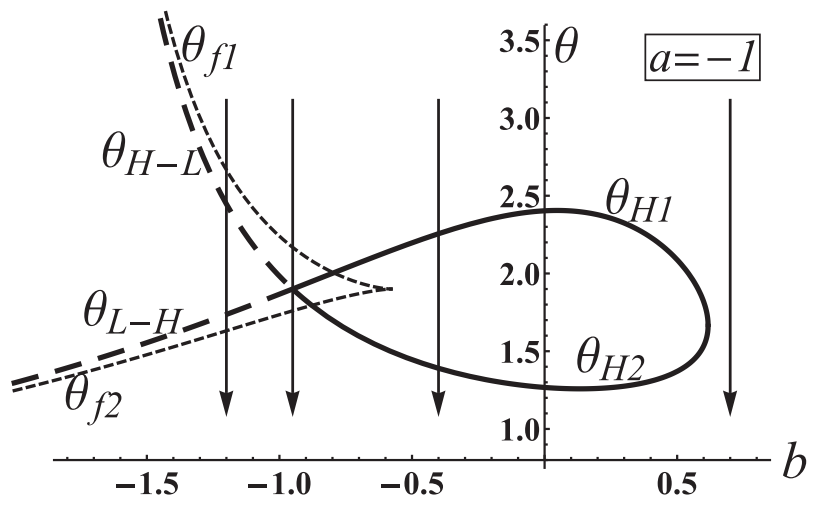

FIG. 8. The control parameter space of the 1-D transport model, for fixed $a<0$, describing L-H transitions by decreasing $\theta$, e.g., the arrows corresponding to the edge steady states visualized in Fig. 7 [(a)-(d) is from left to right]. The short-dashed (cusp shaped) lines correspond to the two fold bifurcations which merge at the cusp bifurcation point (Fig. 6). The Hopf bifurcation curve consists of two parts; the dashed part corresponding to the sharp hysteresis like transitions and the solid curve surrounds the dither regime (Fig. 7).
Fig. 3), which must be the case because they both have the same underlying bifurcation of co-dimension 3 .

\section{CONCLUSION AND DISCUSSION}

In this paper, a general method has been introduced to find a certain bifurcation of co-dimension 3 in systems of both partial differential equations and ordinary differential equations. This is very useful to predict the complex nonlinear dynamical behavior of systems which describe spatial and temporal evolutions simultaneously. This can especially be exploited in systems which exhibit self generated transitions, such as the L-H transition in magnetically confined fusion plasmas. This co-dimension 3 bifurcation combines two different ways of controlling the existence and magnitude of the hysteresis in such systems. One is to merge the two separate fold bifurcations (which cause the separate L-H and H-L transitions), eventually leading to smooth transitions. The other way changes the two stable stationary states (L-mode and H-mode) into unstable stationary states leading to a stable limit cycle solution of the system, resulting in dithering-like transitions. Therefore, this bifurcation is particularly useful for L-H transition models, which must describe these observed transition phenomena. Thus, finding this bifurcation of co-dimension 3 in any dynamical system makes that system a candidate for a $\mathrm{L}-\mathrm{H}$ transition model. The other way around if this bifurcation is not present in a model for the L-H transition, it is not capable of describing all transition phenomena.

Besides this global criterium for L-H transitions, additional threshold criteria for the $\mathrm{L}-\mathrm{H}$ and $\mathrm{H}-\mathrm{L}$ transitions follow directly from the bifurcation analysis in terms of all relevant parameters incorporated in the model. For the example of Zohm's model, ${ }^{8}$ this leads to the physical control parameters nicely combined in the single bifurcation parameter $\theta$ [defined in Eq. (46)]. So, as is expected, increasing the heating power will lead to increasing the heat flux coming from the core, $q_{-\infty}$, and with that it decreases $\theta$ towards its H-mode value. Remarkably, however, this model predicts that increasing the particle flux has the opposite effect of the heating power. This may be interpreted as just extra cooling of the plasma due to these extra particles. This is not observed probably because even in neutral beam heated plasmas, it is hard to increase the particle flux without heating the plasma, also the beneficial effect of extra momentum and flow due to these extra particles is not taken into account in this model. Furthermore, it is noted that many other parameters play a role in the L-H transition such as the edge gradient lengths determined by scrape-off layer physics. Additionally, the exact region of parameter space where dithering transitions are predicted is found due to the bifurcation analysis, as is plotted in Fig. 8.

The usefulness of this new method for future work, is that it is a tool which can be used to compare different L-H transition mechanisms with one another. This can be done in several ways, one is to do the bifurcation analysis on a completely different model and see which other parameters will lead to the different transitions. The other is to incorporate new mechanisms into the transport model of Zohm and see 
how these influence the bifurcating behavior of the model. For instance, a more physics based description of the diffusivity and conductivity can be incorporated, if this mechanism would, for instance, pull the fold bifurcations further apart, then it will probably be a lot easier to go deep into $\mathrm{H}$-mode. The size of the transport barrier and its parametric dependencies can also be determined, because this 1-D system also describes spatial transitions from the L-mode core to the $\mathrm{H}$-mode edge in the region where multiple stationary states are allowed. Another possibility is to see how several mechanisms (as discussed in Sec. IV) influence the parameters of the function $G(Z)$. The entire system can also be extended to incorporate extra dynamical degrees of freedom, such as, for instance, the evolution of the turbulence level in combination with the zonal flows ${ }^{19-21}$ and/or geodesic acoustic modes. ${ }^{22,23}$

\section{ACKNOWLEDGMENTS}

This work, supported by the European Communities under the contract of Association between EURATOM/ FOM, was carried out within the framework of the European Fusion Programme with financial support from NWO. The views and opinions expressed herein do not necessarily reflect those of the European Commission. This work is supported by NWO-RFBR Centre-of-Excellence on Fusion Physics and Technology (Grant No. 047.018.002).

${ }^{1}$ F. Wagner, G. Becker, K. Behringer, D. Campbell, A. Eberhagen, W. Engelhardt, G. Fussmann, O. Gehre, J. Gernhardt, G. v. Gierke, G. Haas, M. Huang, F. Karger, M. Keilhacker, O. Klüber, M. Kornherr,
K. Lackner, G. Lisitano, G. G. Lister, H. M. Mayer, D. Meisel, E. R. Müller, H. Murmann, H. Niedermeyer, W. Poschenrieder, H. Rapp, H. Röhr, F. Schneider, G. Siller, E. Speth, A. Stäbler, K. H. Steuer, G. Venus, O. Vollmer, and Z. Yü, Phys. Rev. Lett. 49, 1408 (1982).

${ }^{2}$ F. Wagner, Plasma Phys. Controlled Fusion 49, B1 (2007).

${ }^{3}$ B. A. Carreras, P. H. Diamond, Y. M. Liangs, V. Lebedev, and D. Newman, Plasma Phys. Controlled Fusion 36, A93 (1994), and references therein.

${ }^{4}$ T. Estrada, C. Hidalgo, T. Happel, and P. H. Diamond, Phys. Rev. Lett. 107, 245004 (2011), and references therein.

${ }^{5}$ R. FitzHugh, Biophys. J. 1, 445 (1961).

${ }^{6}$ J. Nagumo, Proc. IRE 50, 2061 (1962).

${ }^{7}$ Y. A. Kuznetsov, Elements of Applied Bifurcation Theory, 2nd ed. (Springer, New York, 1998).

${ }^{8}$ H. Zohm, Phys. Rev. Lett. 72, 222 (1994).

${ }^{9}$ F. R. Gantmacher, The Theory of Matrices (Chelsea, New York, 1959), Vol. 1.

${ }^{10}$ K. H. Burrell, Phys. Plasmas 4, 1499 (1997).

${ }^{11}$ N. Chakrabarti and J. J. Rasmussen, Phys. Plasmas 6, 3047 (1999).

${ }^{12}$ P. H. Diamond, S.-I. Itoh, K. Itoh, and T. S. Hahm, Plasma Phys. Controlled Fusion 47, R35 (2005).

${ }^{13}$ K. H. Burrell, Phys. Plasmas 6, 4418 (1999).

${ }^{14}$ G. S. Xu, B. N. Wan, H. Q. Wang, H. Y. Guo, H. L. Zhao, A. D. Liu, V. Naulin, P. H. Diamond, G. R. Tynan, M. Xu, R. Chen, M. Jiang, P. Liu, N. Yan, W. Zhang, L. Wang, S. C. Liu, and S. Y. Ding, Phys. Rev. Lett. 107, 125001 (2011).

${ }^{15}$ S.-I. Itoh and K. Itoh, Phys. Rev. Lett. 60, 2276 (1988).

${ }^{16}$ S.-I. Itoh, K. Itoh, A. Fukuyama, and Y. Miura, Phys. Rev. Lett. 67, 2485 (1991).

${ }^{17}$ K. Itoh, Plasma Phys. Controlled Fusion 36, A307 (1994).

${ }^{18}$ K. Itoh and S.-I. Itoh, Plasma Phys. Controlled Fusion 38, 1 (1996).

${ }^{19}$ D. del Castillo-Negrete, B. A. Carreras, and V. E. Lynch, Plasma Phys. Controlled Fusion 46, A105 (2004).

${ }^{20}$ E. Jin Kim and P. H. Diamond, Phys. Plasmas 10, 1698 (2003).

${ }^{21}$ M. A. Malkov and P. H. Diamond, Phys. Plasmas 16, 012504 (2009).

${ }^{22}$ G. D. Conway, C. Angioni, F. Ryter, P. Sauter, J. Vicente, ASDEX Upgrade Team, Phys. Rev. Lett. 106, 065001 (2011).

${ }^{23}$ K. Miki and P. H. Diamond, Phys. Plasmas 17, 032309 (2010). 